\title{
Studies of Enzyme Polymorphisms in the Kamuela Population of Drosophila mercatorum. II. Evaluation of Glycolytic Intermediates
}

\author{
R. L. Clark, ${ }^{1}$ G. J. Brewer, ${ }^{1}$ and C. F. Sing ${ }^{1}$ \\ Received 9 Dec. 1981-Final 2 June 1982
}

A simple and effective cryogenic procedure for the extraction of glycolytic intermediates from whole Drosophila has been developed. This procedure gives consistent results when a measure $\left(\mu \mathrm{M} /\right.$ liter $\left./ O D_{260}\right)$ is adopted which corrects for differences in extraction efficiency. Using this measure and a homozygous strain of $\mathrm{D}$. mercatorum, there are no significant differences among extracts for the levels of any of the 15 glycolytic intermediate or energy molecules considered. The profile of means is consistent across experimental designs and instrument types. Coefficients of variation are well below 50\% for most variables. The methodology presented has the statistical power to detect a mean change of 10 to $50 \%$ using an experimental design which requires as few as 32 observations. The estimated energy charge for resting Drosophila from these studies is the expected value of 0.86 .

KEY WORDS: Drosophila mercatorum; glycolytic intermediates; energy charge.

\section{INTRODUCTION}

This is the second in a series of empirical studies undertaken to relate enzyme polymorphism to components of fitness in a natural population of Drosophila. In the first study (Clark et al., 1981) we characterized the level and nature of the enzyme polymorphisms for the Kamuela, Hawaii, population of Drosophila mercatorum. In this study, we turn to the measurement of glycolytic

This work was supported by NIH Grant 5-R01-AG-01804 and Department of Energy Contract $\mathrm{E}(11-1) 2828$.

' Department of Human Genetics, University of Michigan, Ann Arbor, Michigan 48109. 
intermediate phenotypes which are hypothesized to link inherited enzyme variations with components of reproductive fitness. This is the second step in a three-step "level-crossing" strategy designed to characterize the contribution of allelic differences to variability in intervening phenotypes which may contribute to fitness measures. We have chosen to apply this approach to glycolysis and to consider the glycolytic intermediate (GI) pools as intervening phenotypes, because the controlling glycolytic enzymes have typical levels of polymorphism (Clark et al., 1981), the pathway has an important role in energy metabolism, the enzymes involved are specific and well known, and (as documented in this paper) methods for the measurement of GIs have proven adaptable to Drosophila.

A multivariate vector of glycolytic intermediate phenotypes provides additional information beyond that obtainable from separate studies of the individual variables. The response of glycolysis, as a system, to genetic and environmental perturbations is expressed as changes in both the levels of individual glycolytic pools and the relationships among these pools. A number of studies suggest that GI levels may be a physiological response to genetic and environmental stress treatments which are correlated with flight behavior. Sacktor and co-workers (Sacktor and Hurlbut, 1966; Sacktor and WormserShavit, 1966) have reported altered levels of GIs in the blowfly, Phormia regina, during tethered flight. Preliminary work in our laboratory has shown elevated levels of certain intermediates in D. mercatorum in response to the stress induced by tethered flight, work, or cold. Evidence that allelic differences at glycolytic loci may have effects on flight behavior comes from studies of the screwworm fly, Cochliomyia hominivorax, by Bush and Neck (1976) and of Drosophila by O'Brian and Shimada (1974) and Curtsinger and Laurie-Ahlberg (1981). It is our working hypothesis that the effects of allelic differences at glycolytic loci are expressed as phenotypic differences at the physiological level that are correlated with flight behavior characteristics which contribute to reproductive fitness.

It is the purpose of the present study to develop the methodology for acquiring the measurements of the GIs in Drosophila. The ability to measure these phenotypes accurately will enable us to study a link in the chain of causation between genetic variability and variability in components of fitness.

\section{MATERIALS AND METHODS}

Our work to combine genetics and physiology has led us to prefer Drosophila mercatorum as an experimental organism. While there are several advantages to larger organisms such as Phormia regina, that is, single individuals are readily manipulated and may even suffice for an adequate sample for 
glycolytic intermediate analyses, the extensive genetic information available for the Drosophila species gives them a distinct advantage. The species $D$. mercatorum is particularly useful because of its natural ability to reproduce both sexually and asexually. The asexual process (Carson, 1973; Templeton, 1979a) is a form of parthenogenesis in which the virgin females produce viable diploid eggs. The diploid state is restored to the egg by one of several mechanisms. Gamete duplication (postmeiotic duplication of the haploid gamete followed by fusion) is the most frequent. Each progeny arising from this mechanism is a female, homozygous at all loci, and a candidate to establish an all-female clone.

To produce a truly isogenic bisexual stock from a clone, a y chromosome is added by standard genetical methods. A cloned female is crossed to a male from a stock with a recessive visible marker on each chromosome. The $F_{1}$ male is backcrossed to a female of the original clone to produce a progeny which contains a fraction of males identical (except for the y chromosome) to the cloned females. Such males are identified by a test cross to the females of the marker stock. Males so identified are then crossed to a female of the original clone to produce a bisexual stock which is in all other respects identical to the clone. The absence of crossing-over in male Drosophila and the absolute homozygosity of the cloned females enable one to efficiently create such isogenic lines.

Congenic stocks for allelic variants from the Kamuela population are established on the isogenic background by crossing males of the isogenic bisexual stock to females of a variant line and then repeatedly backcrossing males of the isogenic stock to backcross daughters heterozygous at the locus of interest. These methods have enabled us to develop a series of clones and the isogenic and congenic stocks for the variants isolated from the Kamuela population.

The data reported in this paper are from samples of the isogenic bisexual stock $\mathrm{K}_{23^{-0}}$-bi and the congenic stocks $\mathrm{K}_{23}$-o-bi $(\alpha \mathrm{GP}-\mathrm{F})$ and $\mathrm{K}_{23^{-0}}$-bi $(\alpha \mathrm{GP}$ $\mathrm{S})$, all derived from the clone $\mathrm{K}_{23}$-o-im. The letter $\mathrm{K}$ denotes Kamuela, the collection site in Hawaii; the number 23 indicates the 23 rd wild-caught female whose parthenogenetic daughter was the source of the clone $\mathrm{K}_{23}-\mathrm{O}-\mathrm{im}$. The zero indicates the number of cross-backcross cycles (none) carried out to improve the asexual capacity of the stock (Templeton et al., 1976; Templeton, 1979a). The abbreviations bi and im refer to bisexual and impaternate (all-female) stocks, respectively. The designations $\alpha$ GP-F and $\alpha$ GP-S indicate electrophoretic differences, fast and slow, at the locus coding for $\alpha$ glycerophosphate dehydrogenase. The y chromosome in $\mathrm{K}_{23}-\mathrm{O}-\mathrm{bi}$ is derived indirectly from a single male of another Kamuela stock, $\mathrm{K}_{1}-\mathrm{o}-\mathrm{bi}$, via a marked chromosome stock selected to assure the compatibility of the y chromosome with the rest of the genome. 


\section{Fly-Handling Procedure}

The flies are raised in an incubator at $25^{\circ} \mathrm{C}$ on a $12 \mathrm{hr}$ light $/ 12 \mathrm{hr}$ dark cycle. The medium, $75 \mathrm{ml}$ in 6-oz bottles, contains water, cornmeal, molasses, agar, and brewer's yeast at the ratio 240:23:9:1.5:1 (w/v). The medium is supplemented with $0.1 \mathrm{~g}$ live yeast (dry) per bottle. Six milliliters of proprionic acid per $1200 \mathrm{ml}$ of medium is used as a mold inhibitor. A standard culture is initiated with 15 pairs of flies, the parents are removed on the 19th day, and the progeny are collected on the 25th day. Progeny from 12 bottles are pooled and redistributed on fresh unyeasted bottles, $0.5 \mathrm{~g}$ to a bottle, and fasted for 24 hr prior to sacrifice.

\section{Extraction}

The flies which have been fasted for $24 \mathrm{hr}$ are harvested by decanting directly into liquid nitrogen at $-190^{\circ} \mathrm{C}$. Individual flies boil the nitrogen for less than a second and sink immediately to the bottom of the Dewar flask. The bulk of the liquid nitrogen is decanted, leaving the fly sample in the flask in 2 to $3 \mathrm{ml}$ of liquid nitrogen. The sample of flies is then decanted on top of a 3-vol pellet of frozen $3 \mathrm{M} \mathrm{PCA}$ in a tissue homogenizer tube maintained at $-35^{\circ} \mathrm{C}$ to $-50^{\circ} \mathrm{C}$ in a dry ice-acetone bath. A pestle at $-20^{\circ} \mathrm{C}$ is attached to an automatic stirrer and the sample is subjected to 300 passes in three steps. The extract temperature is monitored with a YSI Model $42 \mathrm{SL}$ telethermometer (Yellow Springs Instrument Co., Inc., Yellow Springs, Ohio) and held at between $-8^{\circ} \mathrm{C}$ and $-12^{\circ} \mathrm{C}$. The sample is held for an additional $15 \mathrm{~min}$ in this temperature range to finish enzyme inactivation. At the end of this inactivation step, 3 vol of $1.0 \mathrm{~mm}$ EDTA in water is added $\left(4^{\circ} \mathrm{C}\right)$ and stirred for 30 min. The sample is centrifuged at $5000 \mathrm{~g}$ for $10 \mathrm{~min}$ to remove protein. It is then decanted, divided into aliquots, and frozen at $-90^{\circ} \mathrm{C}$ until assayed. The samples are thawed on the morning of assay and the procedure given in Fig. 1 is followed. A subsample is removed for measurement of the $\mathrm{OD}_{260}$. The remaining sample is neutralized and spun to clarity. ATP, ADP, AMP, and NAD are measured photometrically on this supernatant. A charcoal treatment is necessary prior to fluorometry to remove pigments from the extract that cause high interfering fluorescence and quenching. The temperatures and timing of the extraction procedure are derived from Lowry and Passonneau (1972).

\section{Drosophila Assay Procedures for Intermediates}

Excitation and fluorescence spectra are measured using a Farrand manual spectrofluorometer with a 16-cell turret and spectroprogrammer (Farrand Optical Co., Inc., Commercial Products Division, Valhalla, N.Y.) and a 


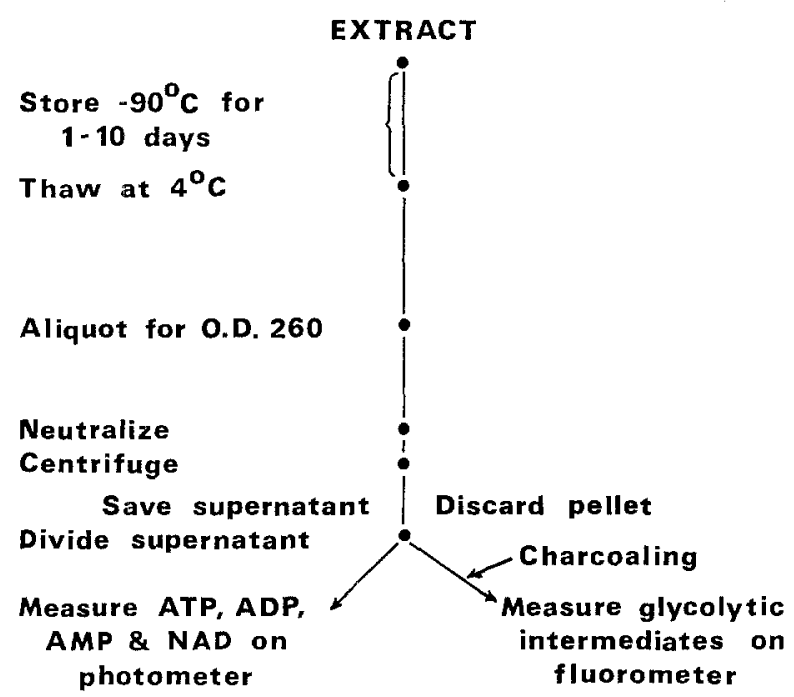

Fig. 1. The flow diagram for processing Drosophila extracts. The $O D$ at $260 \mathrm{~nm}$ is measured on an aliquot of extract. The remaining extract is neutralized with calcium carbonate and centrifuged. Fractions of the supernatant are used for photometric and fluorometric analysis on successive days.

Brinkman Model 2541 potentiometric linear recorder (Goerz Electro, Brinkman Instruments, Westbury, N.Y.). The temperature is kept at a constant $25^{\circ} \mathrm{C}$ in the turret chamber by a circulating water bath (Thermomix Model 1480). The excitation light source is an $85-\mathrm{W}$ mercury arc lamp ( $\mathrm{H} 85 \mathrm{~A} 3 / \mathrm{uv}$; GE Co., Nela Park, Cleveland, Ohio). Ten-millimeter slit widths are used, and the sensitivity range is set to 0.1. A Pyrex glass rod is used as a fluorescent standard for lamp intensity and wavelength calibration; it has an intensity of $30-40$ units at range 0.1 . The gain is set at position 20 . The standard occupies position 0 in the turret and is read before each set of sample readings each time the turret completes a revolution. Any variation in lamp intensity can therefore be adjusted with the gain setting.

Glycolytic intermediates are measured fluorometrically by the conversion of substrate to product in a reaction coupled to the production or consumption of a fluorescent compound, typically NADH or NADPH, with a known stoichiometry. Standard curves of fluorescence versus NADH concentration in appropriate blanks (reaction mixture minus extract) are determined periodically to calibrate fluorescence. The inverse of the slope of the appropriate standard curve ( $\mu \mathrm{M} \mathrm{NADH} /$ liter/chart unit of fluorescence) times the net change in each sample tube (chart units of fluorescence) estimates the glycolytic intermediate concentration ( $\mu \mathrm{M}$ intermediate/liter) since the stoichiometry is known. This value is adjusted for sample dilution and standard- 
ized. Assays of AMP, ADP, ATP, and NAD are carried out on a Gilford 2400 s recording spectrophotometer using quartz semimicro cuvettes (volume, $1.00 \mathrm{ml}$ ). All glassware used in fluorometric determinations is rinsed first in $50 \%$ nitric acid and then in deionized distilled water before use. All solutions with the exception of $1.0 \mathrm{M} \mathrm{MgCl}_{2}$ and $0.9 \mathrm{M} \mathrm{Na}_{2} \mathrm{HAsO}_{4} \cdot 7 \mathrm{H}_{2} \mathrm{O}$ are prepared fresh on the day of assay. The assay procedures are derived from Minakami et al. (1965) as modified by Oelshlegel et al. (1972) unless otherwise noted.

\section{$G 6 P, F 6 P$, and $G 1 P$ - Fluorometric}

This assay is based upon the reduction of cofactor NADP to NADPH by glucose 6-phosphate dehydrogenase and the conversion of G6P to 6-phosphogluconate. The increase in fluorescence due to NADPH is recorded. Subsequent reactions cycle their substrates through this same conversion reaction.

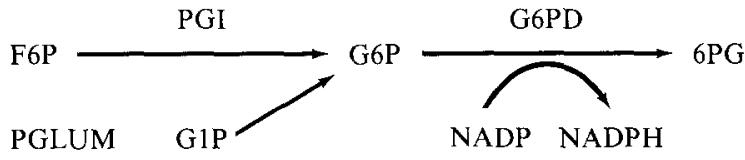

Scheme I

A $1-\mathrm{ml}$ cuvette is loaded with $0.900 \mathrm{ml}$ of PCA blank, $0.100 \mathrm{ml}$ of PCA extract, and $0.005 \mathrm{ml}$ of an NADP solution $(50 \mathrm{mg} / 0.5 \mathrm{ml})$. After baseline readings have been taken, G6P is measured after the addition of $5 \mu \mathrm{l}$ of glucose-6-phosphate dehydrogenase (Boehringer No. 127655, $5 \mathrm{mg} / \mathrm{ml}$ ). Successively, F6P is assayed after the addition of $5 \mu$ phosphoglucose isomerase (Sigma P 8391, 5000 units/1.3 ml), and G1P after the addition of $10 \mu \mathrm{l}$ phosphoglucomutase (Boehringer Mannheim No. 108383, $10 \mathrm{mg} / \mathrm{ml}$ ).

\section{$G A P, D H A P$, and FDP-Fluorometric}

This assay (Neissner and Beutler, 1973) measures the reduction of NAD to NADH in the glyceraldehyde-3-phosphate dehydrogenase-mediated conversion of GAP to 1,3-diphosphoglycerate. Subsequent cycling through this pathway allows us to measure also DHAP and FDP.

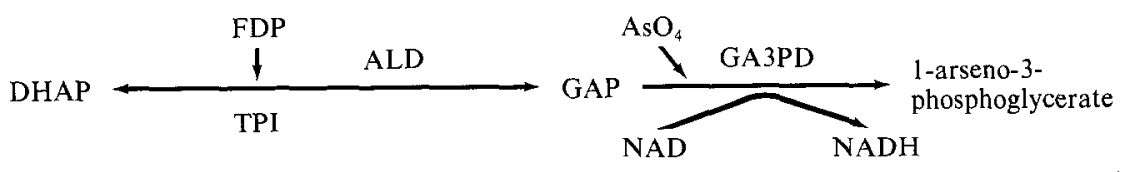


A 1-ml fluorometric cuvette is loaded with $0.8 \mathrm{ml}$ PCA extract, $0.08 \mathrm{ml}$ NAD solution $(50 \mathrm{mg} / \mathrm{ml})$, and $0.16 \mathrm{ml}$ arsenate-mercaptoethanol solution ( $0.36 \mathrm{M}$ sodium arsenate, $0.8 \mathrm{vol} \%$ mercaptoethanol). GAP is measured after the addition of $2 \mu \mathrm{l} \mathrm{GA3PD} \mathrm{(Boehringer} \mathrm{Mannheim} \mathrm{No.} 10568610 \mathrm{mg} / \mathrm{ml}$ ). Following GAP measurement, $12 \mu \mathrm{l}$ additional GA3PD is added to speed the DHAP and FDP measurement reactions. DHAP and FDP are measured after the addition of the following enzymes, respectively: $5 \mu$ l triosephosphate isomerase (Sigma T7877, $10 \mathrm{mg} / \mathrm{ml}$ ) and $10 \mu \mathrm{l}$ aldolase (Sigma A 7145, reconstituted to $10 \mathrm{mg} / \mathrm{ml}$ ).

\section{$\alpha G P$-Fluorometric}

This assay of $\alpha \mathrm{GP}$ is based upon the same reaction (conversion of GAP to 1,3-DPG with a concomitant reduction of NAD) as the above measurements of GAP, DHAP, and FDP. Because Drosophila have higher concentrations of $\alpha$ GP than GAP, DHAP, and FDP, it is necessary to use a much smaller aliquot of extract to avoid quenching. In this assay a $1-\mathrm{ml}$ fluorometric cuvette is loaded with $0.700 \mathrm{ml}$ PCA blank, $0.100 \mathrm{ml}$ PCA extract, $0.080 \mathrm{ml}$ NAD solution $(50 \mathrm{mg} / \mathrm{ml}), 0.071 \mathrm{ml}$ arsenate-mercaptoethanol solution $(0.36 \mathrm{M}$ sodium arsenate, $0.8 \mathrm{vol} \%$ mercaptoethanol), $10 \mu \mathrm{l}$ GA3PD (Boehringer Mannheim No. 105686 10mg/ml), and $5 \mu \mathrm{l} \mathrm{TPI} \mathrm{(Sigma} \mathrm{T7877,} 10 \mathrm{mg} / \mathrm{ml}$ ). After a baseline is established, $10 \mu \mathrm{l} \alpha \mathrm{GPD}$ (Sigma G-6751 1000 units/0.67 $\mathrm{ml}$ ) is added and the reaction recorded.

\section{PYR, PEP, PG2, and PG3-Fluorometric}

This series measures the decrease in fluorescence as NADH is oxidized to NAD in the lactate dehydrogenase-mediated conversion of pyruvate to lactate.

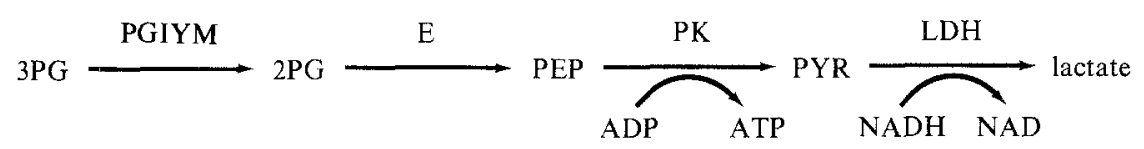

Scheme III

A fluorometric cuvette is loaded with $0.700 \mathrm{ml}$ PCA blank, $0.300 \mathrm{ml}$ PCA extract, $0.030 \mathrm{ml} 1 \mathrm{M} \mathrm{MgCl}_{2}, 10 \mu \mathrm{lADP}(50 \mathrm{mg} / \mathrm{ml})$, and $10 \mu \mathrm{l} \mathrm{NADH}$ $\left(1 \mathrm{mg} / \mathrm{ml}\right.$ in $\mathrm{NaHCO}_{3}-\mathrm{Na}_{2} \mathrm{CO}_{3}, p \mathrm{H} 10.6$, buffer). After a baseline is established, $2 \mu \mathrm{l}$ of $\mathrm{LDH}$ (Sigma L 2500, 25000 units $/ 2.5 \mathrm{ml}$ ) is added. The other intermediates are then measured by adding the following enzymes, respectively; $2 \mu$ l pyruvate kinase (Sigma P 1506, 5000 units $/ 3.2 \mathrm{ml}$ ), $10 \mu \mathrm{l}$ enolase (Sigma E-6126, reconstituted to $10 \mathrm{mg} / \mathrm{ml}$ ), and $10 \mu l$ phosphoglycerate mutase (Boehringer Mannheim No. 108464, $5 \mathrm{mg} / \mathrm{ml}$ ). 
ATP_Photometric (from Minakami et al., 1965)

This assay measures the oxidation of NADH in the coupled reactions mediated by PGK and GA3PD of the conversion 3PG to 1,3-diphosphoglycerate to glyceraldehyde-3-phosphate. Absorbance at $340 \mathrm{~nm}$ is recorded.

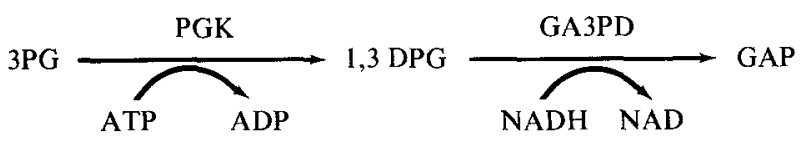

Scheme IV

A quartz semimicro cuvette is loaded with $0.900 \mathrm{ml}$ PCA blank, $0.100 \mathrm{ml}$ PCA extract, $5 \mu 11.0 \mathrm{M} \mathrm{MgCl}_{2}, 0.100 \mathrm{ml} 3 \mathrm{PG}(20 \mathrm{mg} / \mathrm{ml}), 10 \mu \mathrm{l}$ NADH (10 $\mathrm{mg} / \mathrm{ml}$ in $\mathrm{NaHCO}_{3}-\mathrm{Na}_{2} \mathrm{CO}_{3}, p \mathrm{H} 10.6$, buffer), $5 \mu$ mercaptoethanol solution ( $1 \%$ in $\mathrm{H}_{2} \mathrm{O}$ ), and $5 \mu \mathrm{l} \mathrm{GA3PD}$ (Boehringer Manneim No. $10568610 \mathrm{mg} / \mathrm{ml}$ ). Calibrated absorbance is set at 0.5 , and baselines are established. Ten microliters of phosphoglycerate kinase is added (Boehringer Mannheim No. $10843010 \mathrm{mg} / \mathrm{ml})$.

Note: Photometric measurement of ATP, NAD, ADP, and AMP must be carried out on noncharcoaled extract. We found that the adenine and pyridine nucleotides are removed when charcoaling the extract. Also, the eye pigments do not interfere with absorbance readings.

\section{$A D P$ and $A M P$ - Photometric}

This series measures the oxidation of cofactor NADH in the lactate dehydrogenase-mediated conversion of pyruvate to lactate.

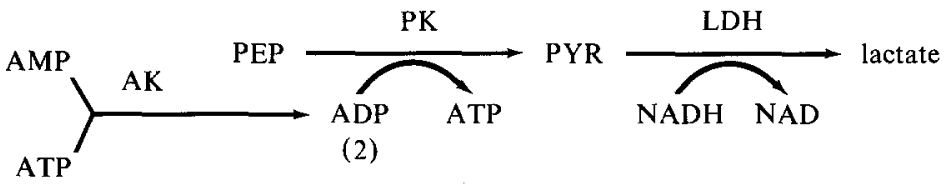

Scheme V

A semimicro quartz cuvette is loaded with $0.900 \mathrm{ml}$ PCA blank, 0.100 PCA extract, $0.030 \mathrm{ml} 1 \mathrm{M} \mathrm{MgCl}_{2}, 0.030 \mathrm{ml}$ phosphoenolpyruvate $[(28$ $\mathrm{mg} / \mathrm{ml})+\operatorname{ATP}(28 \mathrm{mg} / \mathrm{ml})]$, and $0.010 \mathrm{ml} \mathrm{NADH}\left(10 \mathrm{mg} / \mathrm{ml}\right.$ in $\mathrm{NaHCO}_{3}-$ $\mathrm{Na}_{2} \mathrm{CO}_{3}, p \mathrm{H}$ 10.6, buffer). After a baseline is established, $2 \mu \mathrm{l}$ of LDH (Sigma L2500, 25000 units $/ 2.5 \mathrm{ml}$ ) is added. ADP and AMP are assayed by adding, respectively, $2 \mu$ l pyruvate kinate (Sigma P1506 5000 units/3.2 ml) 
and $5 \mu 1$ myokinase (Sigma M3003, $10 \mathrm{mg} / \mathrm{ml}$ ). The calibrated absorbance setting for this series of assays is 0.2 .

\section{NAD-Photometric}

This assay of NAD (Segal et al., 1971) is based upon the reduction of NAD in the alcohol dehydrogenase-mediated conversion of ethanol to acetaldehyde. The $p \mathrm{H}$ of the extract is increased to 8.8 with $\mathrm{K}_{2} \mathrm{CO}_{3}(5 \mathrm{M})$ and the calibrated absorbance is set at 0.2 . A quartz semimicro cuvette is loaded with $0.8 \mathrm{ml}$ ethanol blank ( $30 \mathrm{~mm}$ tetrasodium pyrophosphate, $14.25 \%$ ethanol, $p \mathrm{H} 8.8$ ) and $0.2 \mathrm{ml}$ extract ( $p \mathrm{H} \mathrm{8.8)}$. After a baseline is established, $10 \mu \mathrm{l}$ of alcohol dehydrogenase (Sigma A3263, reconstituted to 3000 units $/ \mathrm{ml}$ ) is added and the reaction recorded.

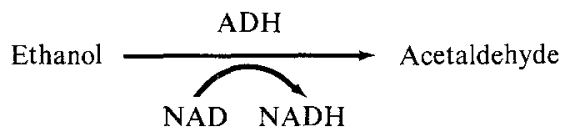

Scheme VI

$O D_{2 \sigma 0}$

Measurements of $\mathrm{OD}_{260}$ are done on a Gilford 2400S in matched sets of quartz semimicro cuvettes. Each cuvette is loaded with $0.900 \mathrm{ml}$ PCA blank and $0.100 \mathrm{ml}$ extract (noncharcoaled) and read against PCA blank at $260 \mathrm{~nm}$.

\section{Experimental Design}

The data reported here were collected on unstressed flies cultured using the standard fly-handling procedures described above. The status "unstressed" denotes that the flies were at rest immediately prior to sacrifice. These data were taken from several different experiments designed (1) to estimate extraneous variability introduced by fly-handling extraction and assay, (2) to estimate the effects of genetic strain and environmental stress (only the unstressed control is reported here) on intermediate levels, or (3) to document the linear regression of intermediate levels on $\mathrm{OD}_{260}$ under the assay conditions described above.

In all cases the genetic variability was controlled by utilizing the isogenic stock $\mathrm{K}_{23}$-o-bi or the closely related congenic stocks $\mathrm{K}_{23}$-o-bi $(\alpha$ GP-F) and $\mathrm{K}_{23}$-o-bi ( $\left.\alpha \mathrm{GP}-\mathrm{S}\right)$. The variability of the fly environment was minimized by the standard culture technique established by experiments which preceded the collection of the data reported here. Possible bottle effects were eliminated by first pooling and then dividing approximately 1800 flies from 12 bottles (each 
strain) into samples of 250 to 300 flies. Each sample was maintained in a bottle containing fresh unyeasted medium for a $24-\mathrm{hr}$ fast prior to extraction.

The design of experiments depended on the instrument available and the complexity of the experimental protocol. In the first set of experiments, design 1 , four aliquots of each of four extracts of $\mathbf{K}_{23}$-o-bi were assayed for each GI variable. We used this design to determine the relative contributions of extraction (extracts) and assay (aliquots) to the total variance of a glycolytic intermediate. In the second set of experiments, design 2, two aliquots of each of eight extracts were assayed for each variable. The object of this design was to contrast two genotypes $\left(\mathrm{K}_{23}-\mathrm{o}-\mathrm{bi}(\alpha \mathrm{GP}-\mathrm{F})\right.$ and $\mathrm{K}_{23}$-o-bi $(\alpha \mathrm{GP}-\mathrm{S})$ in this study) each at two treatment levels. Each treatment strain combination involved two extracts, each with two aliquots. Two aliquots of each of eight extracts of $\mathrm{K}_{23}-\mathrm{O}-\mathrm{bi}$ were assayed for each variable in the study of the regression of $\mathrm{OD}_{260}$ on grams wet weight/liter and the regressions of the glycolytic intermediates on $\mathrm{OD}_{260}$. In these regression experiments the extracts were prepared at four concentrations, with duplication at the extract level.

Both design 1 and design 2 require more assays than there are cuvette positions; consequently multiple runs are required. When pooling data from multiple runs we have taken care not to underestimate aliquot within extract variability. This underestimation occurs due to correlation of errors within a run and, if ignored, could lead to spurious significance in statistical tests. Due to different instrument limitations we have managed the distribution of samples among runs differently for the spectrophotometer and the spectrofluorometer. In the case of the spectrophotometer we have randomized the run errors across extracts which represent the treatment combinations. In the case of the spectrofluorometer all treatment combinations are assayed in the same run. In both cases, the aliquot within extract error is not underestimated due to the correlation of errors which could occur if both aliquots were measured in the same run.

\section{RESULTS AND DISCUSSION}

\section{Choice of Measure}

The glycolytic intermediates were measured as the micromolar concentration per liter of extract $(\mu \mathrm{M} /$ liter $)$ and standardized by division with three alternate measures of the amount of fly extracted. The three measures were grams wet weight per liter ( $g$ wet wt/liter), grams of resolubilized PCAprecipitated protein per liter, and $\mathrm{OD}_{260}$. The first two measures are likely to differ from the third, $\mathrm{OD}_{260}$, if there are differences in extraction efficiencies among extracts. The first two reflect the amount of fly intended for extraction, the weighed amount, but do not vary with the extraction efficiency as $\mathrm{OD}_{260}$ 
does. For this reason, $\mu \mathrm{M} /$ liter $/ \mathrm{OD}_{260}$ is a potentially superior measure of GIs. It is also a convenient metric, in that an aliquot of extract may be used to measure the quantity of fly at a time subsequent to extraction. This is a particularly relevant advantage if a fly-handling procedure, such as weighing, is likely to alter GI pools.

The realization of the potential superiority of the measure $\mu \mathrm{M} /$ liter/ $\mathrm{OD}_{260}$ depends on the demonstration that the $\mathrm{OD}_{260}$ and GIs are released in the same proportion over the range of extraction efficiencies encountered. The first characteristic is demonstrated in Fig. 2 by the linear regression of $O D_{260}$ on $\mathrm{g}$ wet wt/liter. Approximately $97 \%$ of the variability in fly weight is predicted by the $\mathrm{OD}_{260}$. The extractions of GIs and $\mathrm{OD}_{260}$ in fixed proportions are demonstrated by the linear regressions summarized in Table I. We have pooled the photometric data from nine experiments $(N=92)$ but present the fluorometric data separately for two experiments $(N=16$ in each) to exemplify the repeatability of the results. In general, the linear regressions are significantly different from zero except for those intermediates at the lowest concentrations. There were no significant second- or third-order regression effects.

Design 1 resulted in significant differences among extracts within strains for glycolytic intermediates expressed as $\mu \mathrm{M} /$ liter of extract. These differences in GI measures remained when the data were expressed as $\mu \mathrm{M} / \mathrm{g}$ PCA-precipitated protein or $\mu \mathrm{M} / \mathrm{g}$ wet wt but were removed when expressed

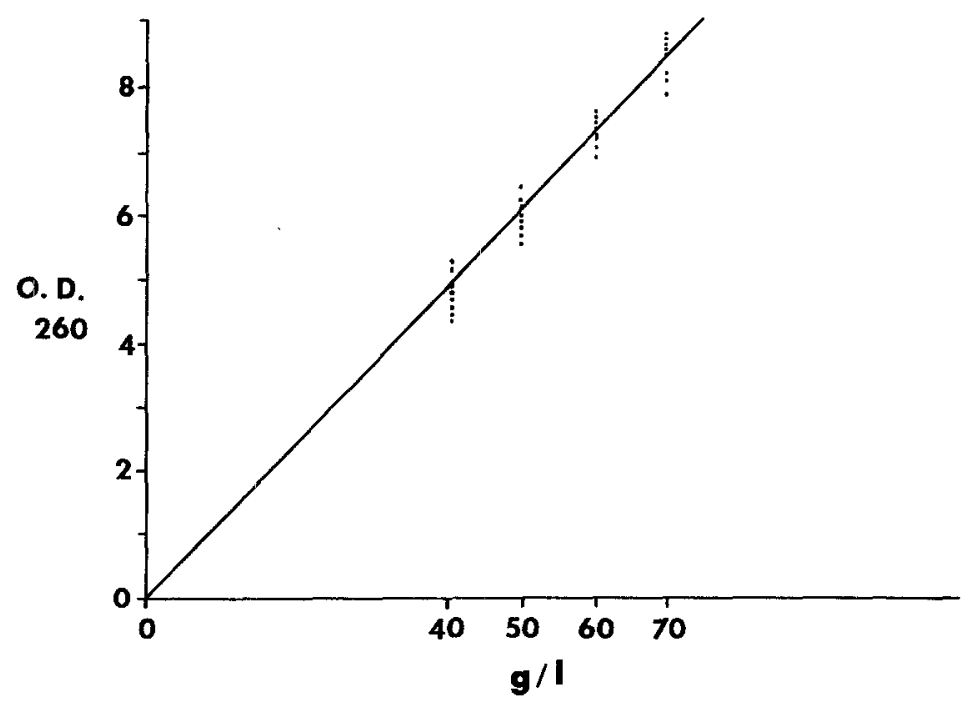

Fig. 2. The linear regression of $O D$ at $260 \mathrm{~nm}$ on grams wet weight of flies per liter $(\beta=0.121)$. Thirty-two observations over four concentrations in our working range are graphed. The $O D$ at $260 \mathrm{~nm}$ predicts $97 \%$ of the variability in grams per liter. 


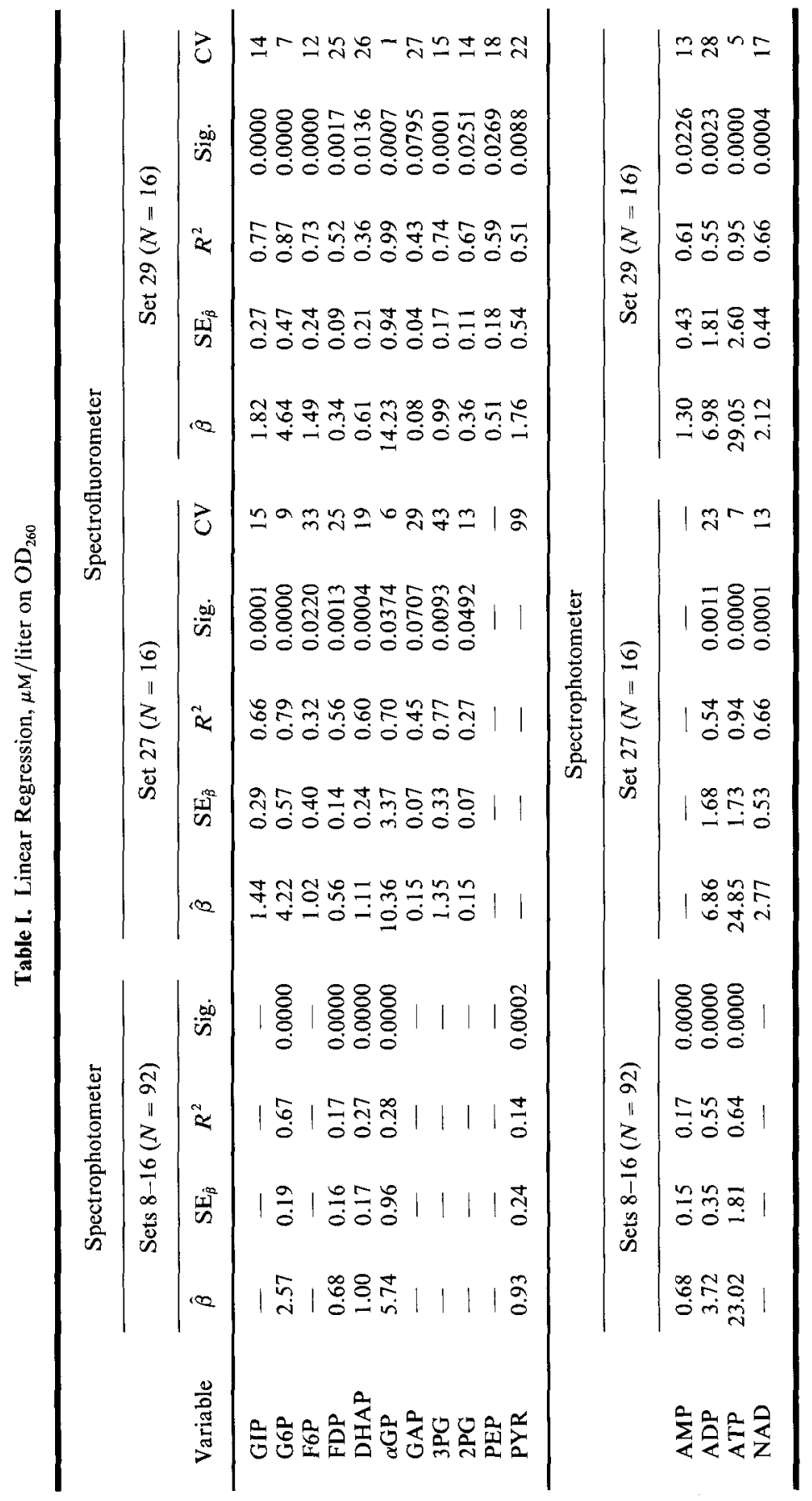


as $\mu \mathrm{M} /$ liter $/ \mathrm{OD}_{260}$. We interpret the $\mu \mathrm{M} /$ liter of extract differences as arising from differences in extraction efficiency which are standardized by the OD at $260 \mathrm{~nm}$. In addition, a comparison of the linear regressions of $\mu \mathrm{M} \mathrm{GI} /$ liter of extract on $\mathrm{OD}_{260}$ and $\mathrm{g}$ wet wt/liter reveals slightly higher $R^{2}$ values for the regression on $\mathrm{OD}_{260}$. For reasons of the convenience, adjustment for differences in extraction efficiency, and improved regression, we find the $\mathrm{OD}_{260}$ to be a superior measure of fly quantity in our extraction procedure.

\section{GI Means and CVs}

The means and CVs for 146 measurements of each glycolytic intermediate on aliquots of 68 extracts of $D$. mercatorum are presented in Table II. While there are small differences in scale, the rank order of the means is consistent

Table II. A Summary of Pool Sizes and Coefficients of Variation for Resting Flies

\begin{tabular}{|c|c|c|c|c|c|c|}
\hline \multirow[b]{3}{*}{ Variable } & \multirow{2}{*}{\multicolumn{2}{|c|}{$\begin{array}{c}\text { Design } 1 \\
\frac{\text { Spectrophotometer }}{(N=16 ;} \\
\left.\mu \mathrm{M} / \text { liter } / \mathrm{OD}_{260}\right)\end{array}$}} & \multicolumn{4}{|c|}{ Design 2} \\
\hline & & & \multicolumn{2}{|c|}{$\begin{array}{l}\text { Spectrophotometer } \\
\qquad(N=48 \\
\left.\mu \mathrm{M} / \text { liter } / \mathrm{OD}_{260}\right)\end{array}$} & \multicolumn{2}{|c|}{$\begin{array}{l}\text { Spectrofluorometer } \\
\qquad(N=82 ; \\
\left.\mu \mathrm{M} / \text { liter } / \mathrm{OD}_{260}\right)\end{array}$} \\
\hline & $\bar{X}$ & $\mathrm{CV}^{a}$ & $\bar{X}$ & $\mathrm{CV}^{b}$ & $\bar{X}$ & $\mathrm{CV}^{b}$ \\
\hline GIP & - & - & - & - & 1.05 & 21 \\
\hline G6P & 2.21 & 9 & 1.83 & 16 & 3.27 & 18 \\
\hline F6P & - & - & - & - & 1.04 & 28 \\
\hline FDP & 0.45 & 38 & 0.56 & 30 & 0.42 & 23 \\
\hline DHAP & 0.81 & 24 & 0.64 & 38 & 0.75 & 18 \\
\hline$\alpha \mathrm{GP}$ & 7.50 & 16 & 6.19 & 23 & 11.06 & 10 \\
\hline GAP & - & - & - & - & 0.11 & 10 \\
\hline 3PG & - & - & - & - & 0.88 & 23 \\
\hline $2 P G$ & - & - & - & - & 0.21 & 74 \\
\hline PEP & - & - & - & - & 0.24 & 36 \\
\hline \multirow[t]{2}{*}{ PYR } & 0.83 & 40 & 0.60 & 46 & 1.26 & 31 \\
\hline & \multicolumn{6}{|c|}{ Spectrophotometer } \\
\hline $\mathrm{OD}_{360}$ & 8.69 & 7 & 6.86 & 19 & 9.40 & 8 \\
\hline NAD & - & - & - & - & 2.53 & 14 \\
\hline AMP & 0.79 & 22 & 0.42 & 45 & 0.72 & 61 \\
\hline ADP & 3.70 & 12 & 3.79 & 12 & 5.65 & 20 \\
\hline ATP & 24.23 & 7 & 23.51 & 13 & 20.95 & 14 \\
\hline ATP/ADP & 6.59 & 8 & 6.30 & 19 & 4.07 & 24 \\
\hline ATP/AMP & 31.40 & 20 & 65.13 & 143 & 44.76 & 79 \\
\hline Adenylate & 28.71 & 6 & 27.69 & 11 & 27.98 & 15 \\
\hline Energy charge & 0.91 & 1 & 0.92 & 1 & 0.86 & 6 \\
\hline
\end{tabular}

${ }^{a}$ Attributable to series, extract, and aliquot within extract.

${ }^{b}$ Attributable to extract and aliquot within extract differences. 
across experimental design and instrument types. Most of the intermediates are measured with sufficient precision by the methods we have developed to detect differences of 10 to $50 \%$ change in the mean GI concentration. A discussion of the statistical power to detect alterations in individual GI concentrations is given below. The intermediates, GAP, 2PG, and PEP, are present in whole fly extracts at low concentrations which yield a change in fluorescence in the range of the background. This contributes to relatively large coefficients of variation. The improvement of the estimation of these intermediates will depend upon a significant reduction in background fluorescence relative to the response in fluorescence due to the glycolytic intermediate.

Coefficients of variability are a function of the experimental design. The CVs in design 1 include variability due to extract, series, extract by series interaction, and aliquots. Variance components were estimated from the analysis of variance (Snedecor and Cochran, 1980). For the 8 intermediates reported under design 1 , on the average, the extract effect determines $7 \%$ of the total phenotypic variance, with a range from 0 to $28 \%$. The average aliquot effect on variance for the same intermediates is $69 \%$, with a range from 28 to $100 \%$. The combined effect of series and the extract by series interaction on the total variance is small by comparison and not statistically significant at the 0.05 level of probability. The CVs under design 1 indicate that GIs can be measured with acceptable precision on whole fly extracts of $D$. mercatorum.

The CVs for design 2 are a measure of the variability of duplicate extracts within runs and are an indication of our ability to make and measure extracts repeatably. A run is a session at the instrument in which a group of concurrent measurements is made on a set of extracts. Here again, the CVs are acceptable for most variables.

The Drosophila extracts contain significant amounts of biological substances which fluoresce over a range of excitation wavelengths overlapping the excitation wavelength for NADH. These fluorescing substances can be removed with a charcoal treatment but this also removes AMP, ADP, ATP, and NAD. Consequently these four substances are still measured on the spectrophotometer. The other intermediates are measured with marginally better CVs on the spectrofluorometer if the extract is charcoal treated.

In Table III we have compared the means and CVs (extracts) for our measures of glycolytic intermediates with those of Sacktor and co-workers (Sacktor and Hurlbut, 1966; Sacktor and Wormser-Shavit, 1966) made on Phormia regina, the blowfly. The blowfly resting means were estimated from the zero-time points of the figures of Sacktor and co-workers. For comparative purposes the $D$. mercatorum means are converted from $\mu \mathrm{M} /$ liter $/ \mathrm{OD}_{260}$ to $\mu \mathrm{M} / \mathrm{g}$ wet wt using the equality, $1 \mathrm{~g}$ wet wt/liter equals $0.121 \mathrm{OD}$ units, estimated from the regression of $\mathrm{OD}_{260}$ on $\mathrm{g}$ wet wt/liter for the D. mercatorum data (Fig. 2). The Drosophila data are for whole flies, while the Phormia 
Table III. Comparisons of Means and CV (Extracts), $\mu \mathrm{M} / \mathrm{g}$ wet wt

\begin{tabular}{|c|c|c|c|c|}
\hline \multirow[b]{3}{*}{ Variable } & \multirow{2}{*}{\multicolumn{2}{|c|}{$\begin{array}{c}\begin{array}{c}\text { Drosophila mercatorum } \\
\text { whole fly extracts }(N=82)\end{array} \\
\text { Spectrofluorometer }\end{array}$}} & \multirow{2}{*}{\multicolumn{2}{|c|}{$\begin{array}{c}\text { Phormia regina }{ }^{a} \\
\text { thorax extracts }(N=39-157) \\
\text { Spectrophotometer }\end{array}$}} \\
\hline & & & & \\
\hline & Mean & $\mathrm{CV}$ & Mean & $\mathrm{CV}$ \\
\hline G6P & 0.40 & 18 & 0.18 & 25 \\
\hline F6P & 0.13 & 28 & 0.05 & 50 \\
\hline FDP & 0.05 & 23 & 0.15 & 20 \\
\hline DHAP & 0.09 & 18 & 0.14 & 20 \\
\hline$\alpha \mathrm{GP}$ & 1.34 & 10 & 1.50 & 15 \\
\hline GAP & 0.01 & 41 & 0.03 & 50 \\
\hline 3PG & 0.11 & 23 & 0.10 & 20 \\
\hline $2 \mathrm{PG}$ & 0.03 & 74 & 0.04 & 45 \\
\hline PEP & 0.09 & 36 & 0.05 & 30 \\
\hline \multirow[t]{2}{*}{ PYR } & 0.15 & 31 & 0.20 & 32 \\
\hline & \multicolumn{4}{|c|}{ Spectrophotometer } \\
\hline AMP & 0.09 & 61 & 0.12 & 25 \\
\hline $\mathrm{ADP}$ & 0.68 & 20 & 1.50 & 16 \\
\hline ATP & 2.53 & 14 & 7.00 & 11 \\
\hline Energy charge $^{b}$ & 0.86 & 6 & 0.90 & \\
\hline
\end{tabular}

${ }^{a}$ From Sacktor and Hurlbut (1966) and Sacktor and Wormser-Shavit, (1966); at least 39 and a maximum of 157 extracts.

${ }^{b}$ Atkinson (1977).

data are for thoraxes. This difference in tissues extracted may account for the generally higher means in the Phormia data, particularly the ATP and ADP levels, since it is likely that the resting flight muscle is metabolically more active, on a per-gram basis, than the whole fly. The higher values of G6P and F6P in the Drosophila data may reflect a species difference in the pool sizes of these intermediates or a greater recovery of upper-pathway intermediates in Drosophila due to the more rapid temperature drop of this much smaller fly upon liquid nitrogen emersion. Given the many possible sources of difference, the Phormia and Drosophila data are remarkably similar.

The coefficients of variation, based on extract variability, for the Drosophila mercatorum data measured on the spectrofluorometer and the Phormia regina data (Sacktor and Hurlbut, 1966; Sacktor and WormserShavit, 1966) measured on the spectrophotometer are also presented in Table III. With a few exceptions, these results are roughly equivalent and indicate that we are measuring most of the glycolytic intermediates with a precision that is comparable to that of published values.

In Table IV we have indicated the differences in treatment means which 
Table IV. Percentage Change in Resting Mean Detectable for a Given Sample Size ${ }^{a}$

\begin{tabular}{|c|c|c|c|c|c|c|}
\hline \multirow[b]{2}{*}{ Variable } & \multicolumn{3}{|c|}{$\alpha=0.05$} & \multicolumn{3}{|c|}{$\alpha=0.01$} \\
\hline & $N=4$ & $N=8$ & $N=16$ & $N=4$ & $N=8$ & $N=16$ \\
\hline $\mathrm{OD}_{260}$ & 11 & 7 & 5 & 17 & 10 & 7 \\
\hline GIP & 28 & 18 & 12 & 46 & 28 & 18 \\
\hline G6P & 24 & 16 & 11 & 40 & 24 & 16 \\
\hline F6P & 39 & 25 & 16 & 63 & 37 & 25 \\
\hline FDP & 32 & 20 & 14 & 51 & 31 & 20 \\
\hline DHAP & 24 & 16 & 11 & 40 & 23 & 16 \\
\hline$\alpha \mathrm{GP}$ & 7 & 5 & 3 & 12 & 7 & 5 \\
\hline GAP & 57 & 38 & 29 & 95 & 67 & 38 \\
\hline PG3 & 31 & 20 & 14 & 50 & 29 & 19 \\
\hline PG2 & 100 & 66 & 43 & 160 & 95 & 62 \\
\hline PEP & 49 & 33 & 20 & 77 & 45 & 33 \\
\hline PYR & 42 & 27 & 18 & 68 & 40 & 27 \\
\hline AMP & 84 & 54 & 37 & 135 & 80 & 53 \\
\hline ADP & 28 & 18 & 12 & 45 & 27 & 18 \\
\hline ATP & 19 & 12 & 8 & 30 & 18 & 12 \\
\hline NAD & 19 & 12 & 8 & 30 & 18 & 12 \\
\hline
\end{tabular}

${ }^{a} N=$ number of extracts in each of two treatments; degrees of freedom $=2 N-2$.

we could detect with our methodology, for the indicated variables, sample sizes, and Type I experimental errors. These are calculated from the means and variance of the variables of the data set (Table II, fluorometric) measured as $\mu \mathrm{M} /$ liter $/ \mathrm{OD}_{260}$. It can be seen that for $\alpha=0.05$ and $N$ (each treatment) $=$ 16 , most variables can be measured well enough to detect 10 to $50 \%$ changes in the mean levels.

Figure 3 is a graphic of glycolytic intermediate means from the fluorometric data (design 2) expressed as $\mu \mathrm{M} /$ liter $/ \mathrm{OD}_{260} \pm 2 \mathrm{SD}$ calculated from the variance of extracts within runs (design 2). This represents the 95\% confidence interval for extracts. The adenosine nucleotides, $\mathrm{NAD}$, and $\mathrm{OD}_{260}$ are also included. The intermediates are arranged in their metabolic sequence within glycolysis. The profile of means and the estimated variability of observations about the means summarizes the scale and variability of the intermediates as measured in our laboratory.

\section{CONCLUSIONS}

We have developed an extraction procedure for glycolytic intermediates in Drosophila mercatorum. This procedure leads to consistent results when a measure is adopted which corrects for differences in extraction efficiencies. The unit $\mu \mathrm{M} /$ liter $/ \mathrm{OD}_{260}$ is such a measure. Using this measure, we found no 


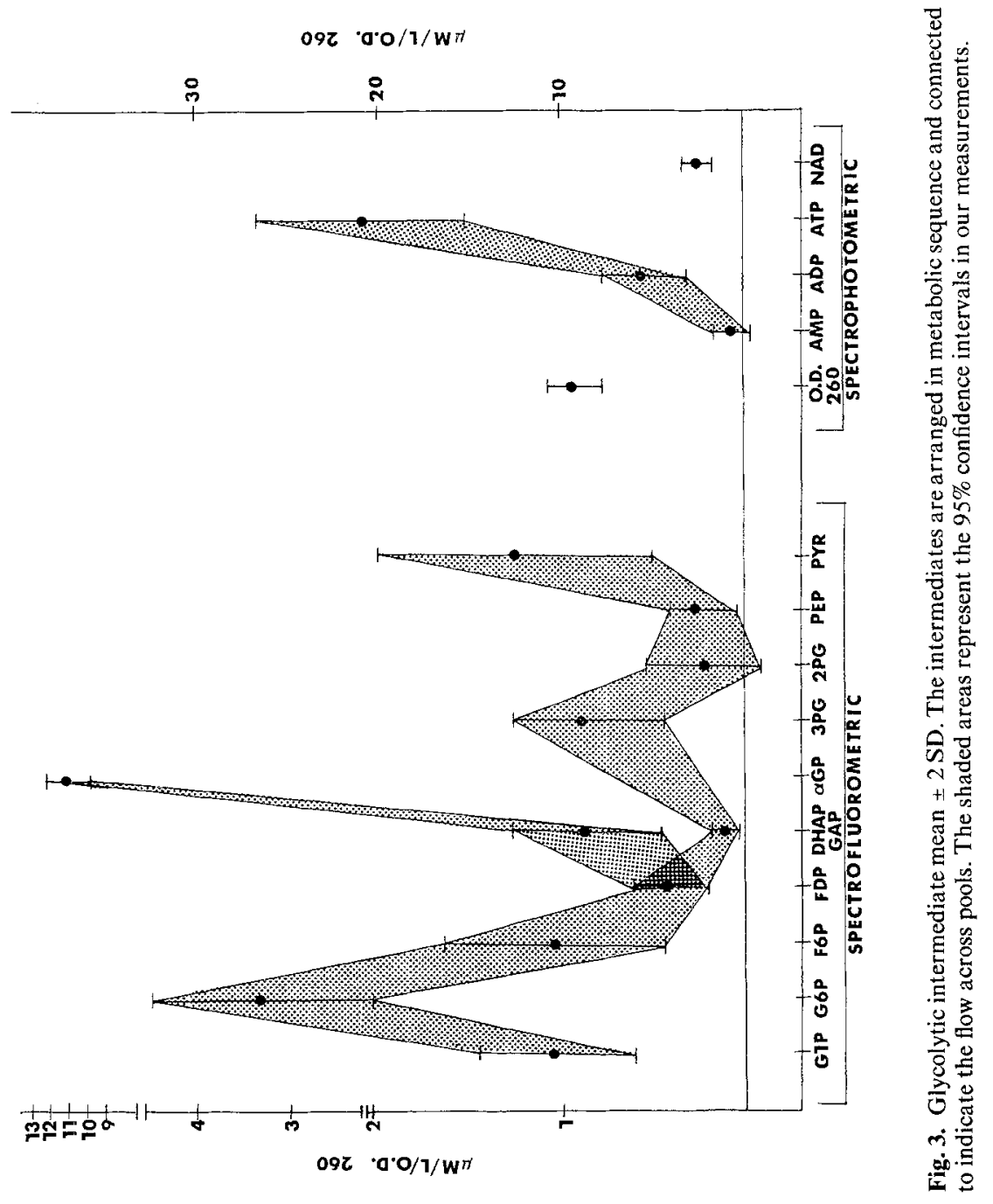


significant differences among extracts for any of the measured metabolic intermediates using the extraction procedure we have developed.

We have compared the means and CVs of the intermediates assayed by the procedure we have developed for Drosophila mercatorum with those obtained for Phormia regina by Sacktor and co-workers (Sacktor and Hurlbut, 1966; Sacktor and Wormser-Shavit, 1966). These measures of ability to extract glycolytic intermediates are roughly equivalent in similar studies. We have estimated that our methodology has the capacity to detect a $10-50 \%$ change in mean level using an experimental design which is convenient.

In summary, the studies reported here document that methods are now available that have an accuracy sufficient to detect relatively small genetic and/or environmental treatment effects on glycolytic intermediate pool levels.

\section{REFERENCES}

Atkinson, D. E. (1977). Cellular Energy Metabolism and Its Regulation, Academic Press, New York and London.

Bush, G. L., and Neck, R. W. (1976). Ecological genetics of the screwworm fly, Cochliomyia hominivora (Diptera: Calliphoridae) and its bearing on the quality control of mass reared insects. Environ. Entomol. 5:821.

Carson, H. L. (1973). The genetic system in parthenogenetic strains of Drosophila mercatorum. Proc. Natl. Acad. Sci. USA 70:1772.

Clark, R. L., Templeton, A. R., and Sing, C. F. (1981). Studies of enzyme polymorphisms in the Kamuela population of $D$. mercatorum. I. Estimation of the level of polymorphism. Genetics 98:597.

Curtsinger, J. W., and Lauric-Ahlberg, C. C. (1981). Genetic variability of flight metabolism in Drosophila melanogaster. I. Characterization of power output during tethered flight. Genetics 98:549.

Lowry, O. H., and Passonneau, J. V. (1972). A Flexible System of Enzymatic Analysis, Academic Press, New York and London.

Minakami, S., Suzuki, C., Saito, T., and Yoshikawa, H. (1965). Studies on erythrocyte glycolysis. I. Determination of the glycolytic intermediates in human erythrocytes. J. Biochem. 58:543.

Neissner, H., and Beutler, E. (1973). Contamination of commercially available intermediates of the glycolytic pathway. Experientia 29:268.

O'Brian, S. J., and Shimada, Y. (1974). The $\alpha$-glycerophosphate cycle in Drosophila melanogaster. IV. Metabolic, ultrastructural, and adaptive consequences of $\alpha \mathrm{GPDH}-1$ "null" mutations. J. Cell Biol. 63:864.

Oelshlegel, F. J., Brewer, G. J., Penner, J. A., and Schoomaker, E. B., (1972). Enzymatic mechanisms of red cell adaptation to anemia. In Brewer, G. J. (ed.), Hemoglobin and Red Cell Structure and Function, Plenum Press, New York, Vol. 28, pp. 377-396.

Sacktor, B., and Hurlbut, E. C. (1966). Regulation of metabolism in working muscle in vivo. II. Concentrations of adenine nucleotides, arginine phosphate, and inorganic phosphate in insect flight muscle during flight. J. Biol. Chem. 241:632.

Sacktor, B., and Wormser-Shavit, E. (1966). Regulation of metabolism in working muscle in vivo. I. Concentration of some glycolytic, tricarboxylic acid cycle, and amino acid intermediates in insect flight muscle during flight. J. Biol. Chem. 241:624. 
Segal, G. B., Feig, S. A., Baehner, R. L., and Nathan, D. G. (1971). Fluorometric analysis of glycolytic intermediates and pyridine nucleotides in peripheral blood cells. J. Lab. Clin. Med. 78:969.

Snedecor, G. W., and Cochran, W. G. (1980). Statistical Methods, 7th ed., Iowa State University Press, Ames.

Templeton, A. R. (1979a). The unit of selection in Drosophila mercatorum. II. Genetic revolution and the origin of coadapted genomes in parthenogenetic strains. Genetics 92:1265.

Templeton, A. R. (1979b). The parthenogenetic capacities and genetic structures of sympatric populations of Drosophila mercatorum and Drosophila hydei. Genetics 92:1283.

Templeton, A. R., Carson, H. L., and Sing, C. F. (1976). The population genetics of parthenogenetic strains of Drosophila mercatorum. II. The capacity for parthenogenesis in a natural, bisexual population. Genetics 82:527. 